\title{
From asymmetrical to the nonreciprocal isolator using time-varying metasurfaces
}

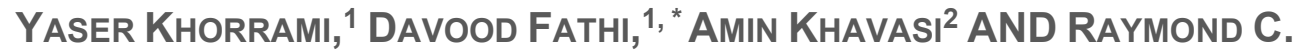 \\ RUMPF $^{3}$ \\ ${ }^{I}$ Department of Electrical and Computer Engineering, Tarbiat Modares University, Tehran, Iran \\ ${ }^{2}$ Department of Electrical Engineering, Sharif University of Technology, Tehran, Iran \\ ${ }^{3}$ EM lab, Department of Electrical and Computer Engineering, University of Texas at El Paso, El Paso, \\ Texas 79968, USA \\ *Corresponding author:d.fathi@modares.ac.ir
}

\begin{abstract}
We present an emulation design method for converting asymmetric isolators to nonreciprocal ones using time-varying metasurfaces. To illustrate the model, we design a structure using a combination of the photonic crystal $(\mathrm{PhC})$ and time-varying metasurface. Moreover, we propose a general approach for numerical analysis of the time-modulated proposed structure using the extension of the transfer matrix method (TMM) which consists of working through the device one layer at a time and calculating an overall transfer matrix including the time-variation of the permittivity and permeability in each layer. Also, we use an optimization algorithm that is less used in the field of electromagnetism but is suitable for fast and accurate parameter optimization. The results show that the proposed method, using pure time-varying metasurfaces which cannot prepare full nonreciprocity alone, is a promising procedure for breaking the Lorentz reciprocity in the general isolator system as well as maintaining the previously asymmetric designed structure.
\end{abstract}

Keywords: Nonreciprocal; asymmetrical; isolator; spacetime; metasurface.

\section{Introduction}

Time-dependent metasurfaces are now widely proposed in optical applications (Caloz and Deck-Léger 2020; Chamanara et al. 2019; Taravati and Eleftheriades 2019; Pendry 2008; Cui et al. 2019). These structures are either purely temporal or spatiotemporal used (Pacheco-Peña and Engheta 2020; Zang et al. 2019; Taravati 2018). Combining them with spatial manipulation of the surface increases the ability to control the properties of light and, more importantly, ensures nonreciprocity properties (Shaltout et al. 2015). But in the time-gradient metasurface, despite the lack of conservation of the energy and the vertical component of the momentum, due to the conservation of the tangential components of the momentum, the nonreciprocity is not provided (Wu and Grbic 2020; Xiao et al. 2014). So far, some ideas have been given for this subject so that by changing the phase, it is possible to move from purely time-modulated metasurfaces to nonreciprocal applications (Chegnizadeh et al. 2020; Sedeh et al. 2020).

On the other hand, asymmetric structures are widely proposed in many optical applications (Ba et al. 2019; Tang et al. 2016; Ling et al. 2017). Although asymmetric structures cannot provide the property of nonreciprocal (Jalas et al. 2013), in many cases, they are expediter (Khorrami et al. 2020; Xu and Lezec 2014). Two common candidates to the design of the asymmetrical one-way transmitters or isolators are grating (Li et al. 2019; Stolarek et al. 2013; Cakmakyapan et al. 2010; Zhu et al. 2018; Xu et al. 2018) and PhC (Serebryannikov et al. 2016; Maystre 2001; Blankrot and Heitzinger 2019; Bor et al. 2018). In this paper, we try to emulate a structure with the capability of nonreciprocity by using the combination of purely time-modulated metasurfaces and $\mathrm{PhC}$ reflector, which in addition to the need to design asymmetric structures, can also have nonreciprocal behavior. The advantage of this method is that it is easy to equip the former asymmetric structures to the nonreciprocity property without the need for a new and time-consuming design, just by adding time-varying metasurfaces to the asymmetric structures. 
Another advantage of this method is that we have avoided spatial manipulations at the metasurface and only temporal changes are sufficient. It should be noted that although our numerical method is simulation, our overall model is emulation in which extended numerical simulations are developed in combination with a promising structure using extraordinary material properties. Therefore, we try to model an approximation of the real system as a way to investigate the field of spacetime metasurfaces.

Temporal changes can be achieved by applying external electrical bias (Salary et al. 2019; Yuanhang et al. 2020; Salary et al. 2018) or other various methods (Shaltout et al. 2019). To perform the model, we have developed the transfer matrix method (TMM) (Khorrami et al. 2020; Salary et al. 2018; Hao and Zhou 2008) to be able to solve the time-varying problem for time-dependent permittivity and permeability. Finally, using numerical simulation by the socalled time-varying TMM or TTMM, we have designed an isolator system combining the real $\mathrm{PhC}$ reflector and unrealistic promising model of time-dependent surfaces. This proposed combination effectively breaks the time-reversal symmetry.

This paper is organized as follows: Section 2 explains the numerical method formulation. Implementation of formulation in the form of the proposed structure is discussed in section 3 . In section 4, the results are discussed and finally, the paper concludes in section 5.

\section{Formulation}

We assume the normalized magnetic field of $\overrightarrow{\widetilde{H}}=-j \sqrt{\mu_{0} / \varepsilon_{0}} \vec{H}$ and convolutional timedependent Maxwell's equation with negative sign convention. Permittivity $\left(\varepsilon=\varepsilon_{r} \varepsilon_{0}\right)$ and permeability $\left(\mu=\mu_{r} \mu_{0}\right)$ are consisted of relative and free space $\left(\varepsilon_{0}\right.$ and $\left.\mu_{0}\right)$ which the latter components are related to each other via the light speed $\left(c=\left(\sqrt{\varepsilon_{0} \mu_{0}}\right)^{-1}\right)$. With considering incident wavevectors of $k_{x, \text { inc }}$ and $k_{y, \text { inc }}$ and temporal frequency of $\omega$, we have Fourier expansion of the fields as:

$$
\begin{aligned}
& \vec{E}_{\{x, y, z\}}(x, y, z, t)=\sum_{u=-\infty}^{+\infty} S_{\{x, y, z\}}(u ; z) e^{-j \psi_{u}(x, y, t)} \\
& \overrightarrow{\tilde{H}}_{\{x, y, z\}}(x, y, z, t)=\sum_{u=-\infty}^{+\infty} U_{\{x, y, z\}}(u ; z) e^{-j \psi_{u}(x, y, t)}
\end{aligned}
$$

Where $\psi_{u}(x, y, t)=k_{x, \text { inc }} x+k_{y, \text { inc }} y-\omega(u) t, \mathrm{~S}$ and $\mathrm{U}$ are phase relation, electric and magnetic Fourier coefficients, respectively. Like original TMM, in comparison to the Fourier modal method (FMM), we don't have any periodicity along $x$ - and $y$-directions and all layers are spatially invariant. In TTMM we define time periodicity as $T(=2 \pi / \Omega)$, where $\Omega\left(=2 \pi f_{m}\right)$ is the modulation frequency with the frequency of $f_{m}$. By considering temporal variations, the periodicity is implemented to relative permittivity as $\varepsilon_{r}(t+u T)=\varepsilon_{r}(t)$, which is applicable for permeability, too. Hence, the periodic optical constants can be defined in the transverse plane as:

$$
\begin{aligned}
& \varepsilon_{r}(x, y, t)=\sum_{u=-\infty}^{+\infty} a_{u} e^{-j \frac{2 \pi}{T} u t} \\
& \mu_{r}(x, y, t)=\sum_{u=-\infty}^{+\infty} b_{u} e^{-j \frac{2 \pi}{T} u t}
\end{aligned}
$$

Also, angular frequency is expanded as $\omega(u)=\omega_{i n c}-\frac{2 \pi}{T} u$ with integer number of $u$. The terms of $k_{x, i n c}, k_{y, i n c}$, and $\omega_{i n c}$ are the wavevectors and angular frequency of the incident wave 
along $x$-, $y$ - and $t$ - directions. Using the Cauchy product of two infinite series $\left(\sum_{i=0}^{\infty} \alpha_{i} \cdot \sum_{i=0}^{\infty} \beta_{i}=\sum_{i=0}^{\infty} \gamma_{i}\right.$ where $\left.\gamma_{i}=\sum_{j=0}^{i} \alpha_{i} \beta_{i-j}\right)$ over Eqs (1)-(4), we have:

$$
\begin{aligned}
& \varepsilon_{r}(x, y, t) * \vec{E}_{\{x, y, z\}}=\sum_{u=-\infty}^{+\infty}\left\{\sum_{s=-\infty}^{+\infty} a_{u-s} S_{\{x, y, z\}}^{(s ; z)} e^{-j \psi_{u}(x, y, t)}\right\} \\
& \mu_{r}(x, y, t) * \overrightarrow{\tilde{H}}_{\{x, y, z\}}=\sum_{u=-\infty}^{+\infty}\left\{\sum_{s=-\infty}^{+\infty} b_{u-s} U_{\{x, y, z\}}^{(s ; z)} e^{-j \psi_{u}(x, y, t)}\right\}
\end{aligned}
$$

By substituting Eqs. (5) \& (6) into Maxwell's equations and differentiation over time, we obtain six equations as:

$$
\begin{aligned}
-j \tilde{k}_{i n c, y} \mathbf{s}_{z}-\frac{d}{d \tilde{z}} \mathbf{s}_{y}=\llbracket \mu_{r} \rrbracket \mathbf{W} \mathbf{u}_{x} & \text { (7) } & -j \tilde{k}_{i n c, y} \mathbf{u}_{z}-\frac{d}{d \tilde{z}} \mathbf{u}_{y}=\llbracket \varepsilon_{r} \rrbracket \mathbf{W} \mathbf{s}_{x} \\
\frac{d}{d \tilde{z}} \mathbf{s}_{x}+j \tilde{k}_{i n c, x} \mathbf{s}_{z}=\llbracket \mu_{r} \rrbracket \mathbf{W} \mathbf{u}_{y} & \text { (8) } & \frac{d}{d \tilde{z}} \mathbf{u}_{x}+j \tilde{k}_{i n c, x} \mathbf{u}_{z}=\llbracket \varepsilon_{r} \rrbracket \mathbf{W} \mathbf{s}_{y} \\
\tilde{k}_{i n c, x} \mathbf{s}_{y}-\tilde{k}_{i n c, y} \mathbf{s}_{x}=j \llbracket \mu_{r} \rrbracket \mathbf{W} \mathbf{u}_{z} & \text { (9) } & \tilde{k}_{i n c, x} \mathbf{u}_{y}-\tilde{k}_{i n c, y} \mathbf{u}_{x}=j \llbracket \varepsilon_{r} \rrbracket \mathbf{W} \mathbf{s}_{z}
\end{aligned}
$$

Where the matrix form of $S$ and $U$ have represented in bold lowercase letters. The $\mathbf{W}$ consist of diagonal matrix containing the angular frequency $\omega(u)$ components. We have defined the normalized variables for the incident wavenumbers and longitudinal length through $\tilde{k}_{\{\mathrm{inc} ; x, y\}}=$ $c k_{\{\text {inc; } x, y\}}$ and $\tilde{\mathrm{z}}=\mathrm{z} / c$, respectively. Toeplitz matrix of $\llbracket \varepsilon_{r} \rrbracket$ and $\llbracket \mu_{r} \rrbracket$ are made from the Fourier coefficients $a_{u}$ and $b_{u}$, where each element of $(\alpha, \beta)$ in the Toeplitz matrix can be achieved as $a_{u_{\alpha}-u_{\beta}}$ and $b_{u_{\alpha}-u_{\beta}}$. After a few manipulations, we reach double first-order differential compact equations as:

$$
\frac{d^{2}}{d z^{2}}\left[\begin{array}{l}
\mathbf{s}_{x} \\
\mathbf{s}_{y}
\end{array}\right]=\mathbf{P Q}\left[\begin{array}{l}
\mathbf{s}_{x} \\
\mathbf{s}_{y}
\end{array}\right]
$$

where

$$
\begin{aligned}
& \mathbf{P}=\left[\begin{array}{cc}
\tilde{k}_{i n c, x} \llbracket \varepsilon_{r} \rrbracket^{-1} \mathbf{W}^{-1} \tilde{k}_{i n c, y} & \llbracket \mu_{r} \rrbracket \mathbf{W}-\tilde{k}_{i n c, x} \llbracket \varepsilon_{r} \rrbracket^{-1} \mathbf{W}^{-1} \tilde{k}_{i n c, x} \\
\tilde{k}_{i n c, y} \llbracket \varepsilon_{r} \rrbracket^{-1} \mathbf{W}^{-1} \tilde{k}_{i n c, y}-\llbracket \mu_{r} \rrbracket \mathbf{W} & -\tilde{k}_{i n c, y} \llbracket \llbracket \varepsilon_{r} \rrbracket^{-1} \mathbf{W}^{-1} \tilde{k}_{i n c, x}
\end{array}\right] \\
& \mathbf{Q}=\left[\begin{array}{cc}
\tilde{k}_{i n c, x} \llbracket \mu_{r} \rrbracket^{-1} \mathbf{W}^{-1} \tilde{k}_{i n c, y} & \llbracket \varepsilon_{r} \rrbracket \mathbf{W}-\tilde{k}_{i n c, x} \llbracket \mu_{r} \rrbracket^{-1} \mathbf{W}^{-1} \tilde{k}_{i n c, x} \\
\tilde{k}_{i n c, y} \llbracket \mu_{r} \rrbracket^{-1} \mathbf{W}^{-1} \tilde{k}_{i n c, y}-\llbracket \varepsilon_{r} \rrbracket \mathbf{W} & -\tilde{k}_{i n c, y} \llbracket \mu_{r} \rrbracket^{-1} \mathbf{W}^{-1} \tilde{k}_{i n c, x}
\end{array}\right]
\end{aligned}
$$

Eq. (13) must be solved for each time-modulated layer. Fig. 1(a) depicts a schematic of a hypothetical multilayer stack with incident (green), transmitted (blue) and reflected or refracted (red) beams. Each layer can be excited with external electrical bias to create time-modulated permittivity via the change of the carrier density that is injected to the bars (Park et al. 2017). The incident wavevector $\left(k_{i n c}\right)$ tackles with the first layer by elevation $(\theta)$ and azimuth $(\varphi)$ angles and divides to three directional wavevectors. 
(a)

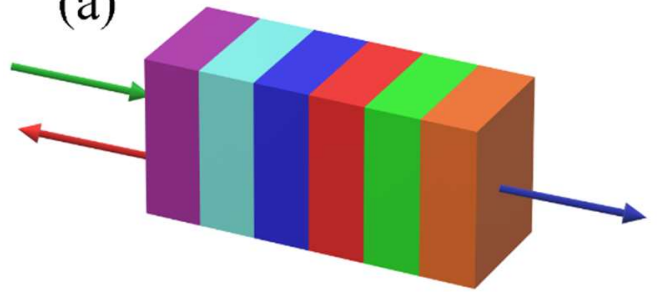

(b)

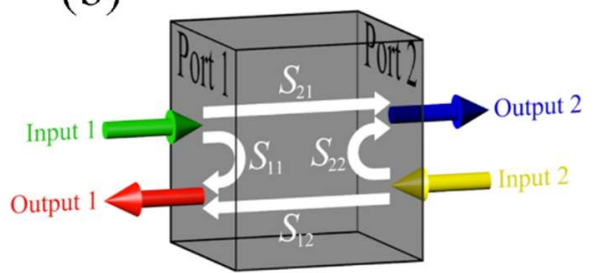

Fig. 1: (a) Temporally modulated multilayer stack. The incident wave (green line) is injected along the $z$ direction and layers are in the $x y$ transverse plan. Refraction and transmission have shown with red and blue line, respectively. (b) Conventional definition of the scattering parameters.

We use a temporal scattering matrix to show the effect of each layer. The TMM consists of working through the device one layer at a time and calculating an overall (global) transfer matrix. In this approach, we add a zero-thickness virtual layer before and after each layer and then try to combine them via the Redheffer star product. To do this, we extend the traditional scattering matrix by considering the variation of angular frequency matrix $\mathbf{W}$ in zero-thickness reflection and transmission side layers. From N-port network theory conventions, scattering parameters of $S_{i j}$ are quantifying the amplitude of the wave exiting port "I" relative to a source injected into port " $\mathrm{j}$ ". That is convention and scattering parameters should be consistent with this convention or it is confusing as shown in Fig. 1(b). The scattering matrices derived and implemented in the literature for semi-analytical methods have abandoned this convention (Rumpf 2011). While not mathematically incorrect, it makes it confusing and inconsistent with how test equipment would measure things. Free space zero thickness air gaps between the layers relate fields that exist outside of the layers. This framework produces symmetric matrices where $S_{11}=S_{22}$ and $S_{12}=S_{21}$ (as scattering matrix elements) for all of the layers composing the multilayer device. This makes the simulation more efficient, faster and consumes less computing cost and memory.

\section{Proposed structure}

We proposed a nonreciprocal isolator consisting of a photonic crystal $(\mathrm{PhC})$ reflector and two time-varying metasurfaces as seen in Fig.2. Recently asymmetrical PhC- based structure using static grating devices has been proposed (Khorrami et al. 2020). As depicted in the cross-section view of Fig. 2, the forward incident plane wave excites the structure in the forward direction. In the same way, the backward incident plane wave excites the structure from the bottom side. By considering PhC solely as depicted in Fig. 3, the diffracted light beam can reach the cylindrical rods and blocked through the $\mathrm{PhC}$ bandgap at some wavelengths. 

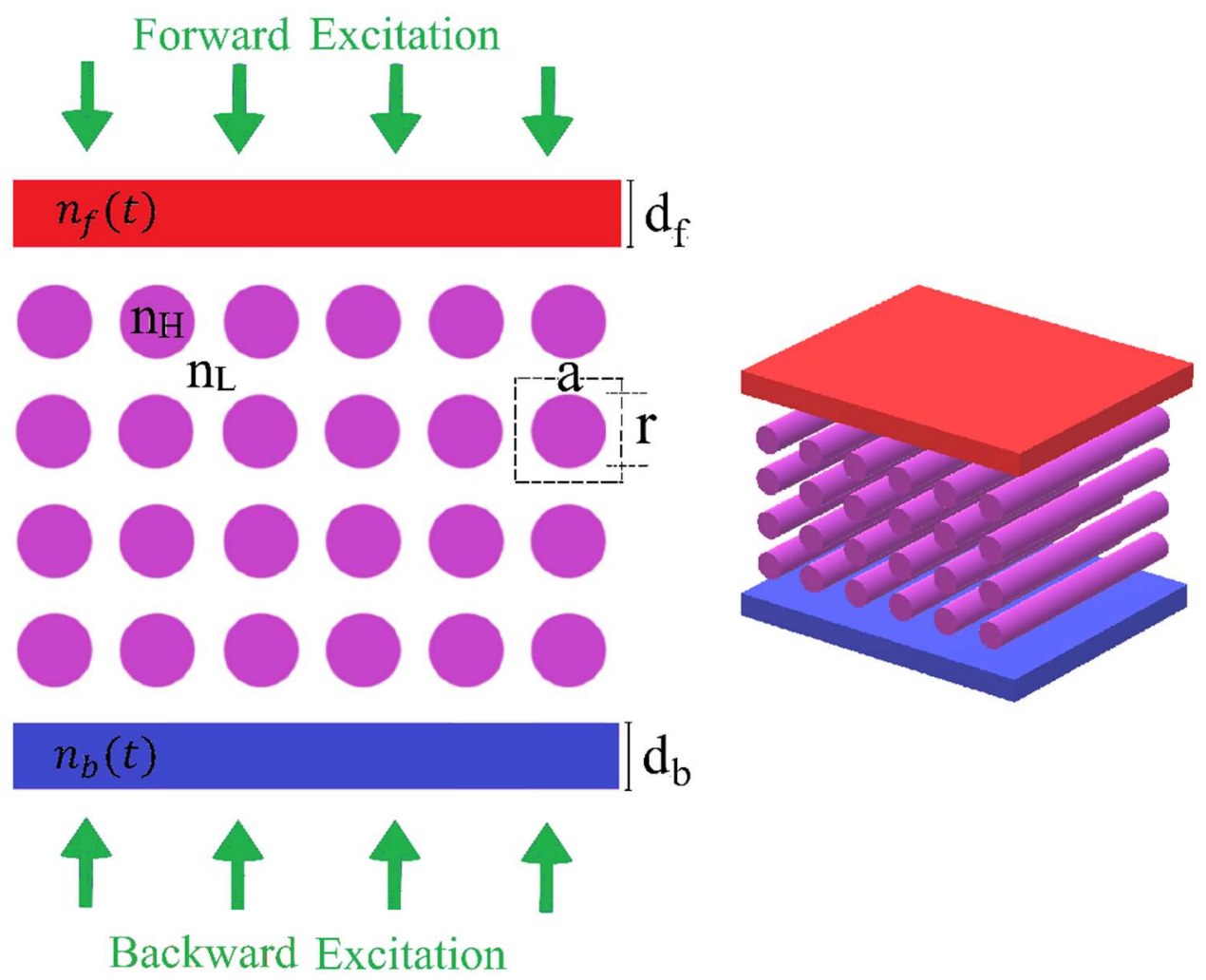

Fig. 2: Cross-section of the isolator using a combination of time-varying metasurfaces and the customized $\mathrm{PhC}$. The time-modulated refractive index and thicknesses of the top and bottom slabs are $n_{f}(t), d_{f}$ and $n_{b}(t), d_{b}$, respectively. Each $\mathrm{PhC}$ rods have a refractive index of $n_{H}$ that is surrounded with $n_{L}$. Square unit cells have a dimension of "a" including rods with an " $r$ " radius. The vertical direction is along the $y-$ direction. The inset is a $3 \mathrm{D}$ schematic of the figure.

To design the structure, we use the cultural algorithm (CA) as reported in (Khorrami et al. 2020), firstly to optimize parameters of the PhC rods, $\{\mathrm{a}, \mathrm{r}\}$, to reach the proper bandgap in the range from 3.23 to $4.25 \mathrm{THz}$, and secondly for optimizing the time-varying slabs. The parameter ' $a$ ' is the length of the square unit cell from the cross-section view including each rod with the radius of ' $r$ '. For the simulation of the $\mathrm{PhC}$ structure, we used the in-house finite difference frequency domain (FDFD) program (Khorrami et al. 2020) to rigorously model the light diffraction in each mesh point over the whole structure. We used the PML boundary condition on both sides of the device along y axis to restrict the structure along with the vertical directions. Both the forward and backward incident waves are normal with the transverse magnetic (TM) polarization. The refractive indices of the rods are assumed as $\mathrm{n}_{\mathrm{H}}=3.5$ surrounded by $\mathrm{n}_{\mathrm{L}}=1.0$. The parameters of $\mathrm{PhC}$ are optimized as $\mathrm{a}=33.14 \mu \mathrm{m}, \mathrm{r}=0.354 \mathrm{a} \mu \mathrm{m}$. To reach a nonreciprocal isolator, we need to design forward and backward time-varying metasurfaces for down- and up-converting the frequency. We used TTMM to simulate the timevarying slab and verified the results with common finite difference time domain (FDTD) methods (Stewart et al. 2018). If we set the input frequency as $f_{0}=3.24 \mathrm{THz}$, the frequency modulation of the system can be set as $f_{m}=34 \mathrm{GHz}$. Using CA, we calculate the exact parameter of the input time-varying slab with a time-dependent permittivity of $\varepsilon_{f}(t)=\varepsilon_{r f}(1+$ $\left.\delta_{\varepsilon} f_{m} t\right)$ as $\varepsilon_{r f}=12.25, \delta_{\varepsilon}=0.27161$ and $d_{f}=28.755 \mu \mathrm{m}$, where $\delta_{\varepsilon}$ is the modulation 
depth of the permittivity. The maximum modulation depth can be achieved in graphene electrooptical modulators (Inampudi et al. 2019; Phare et al. 2015).

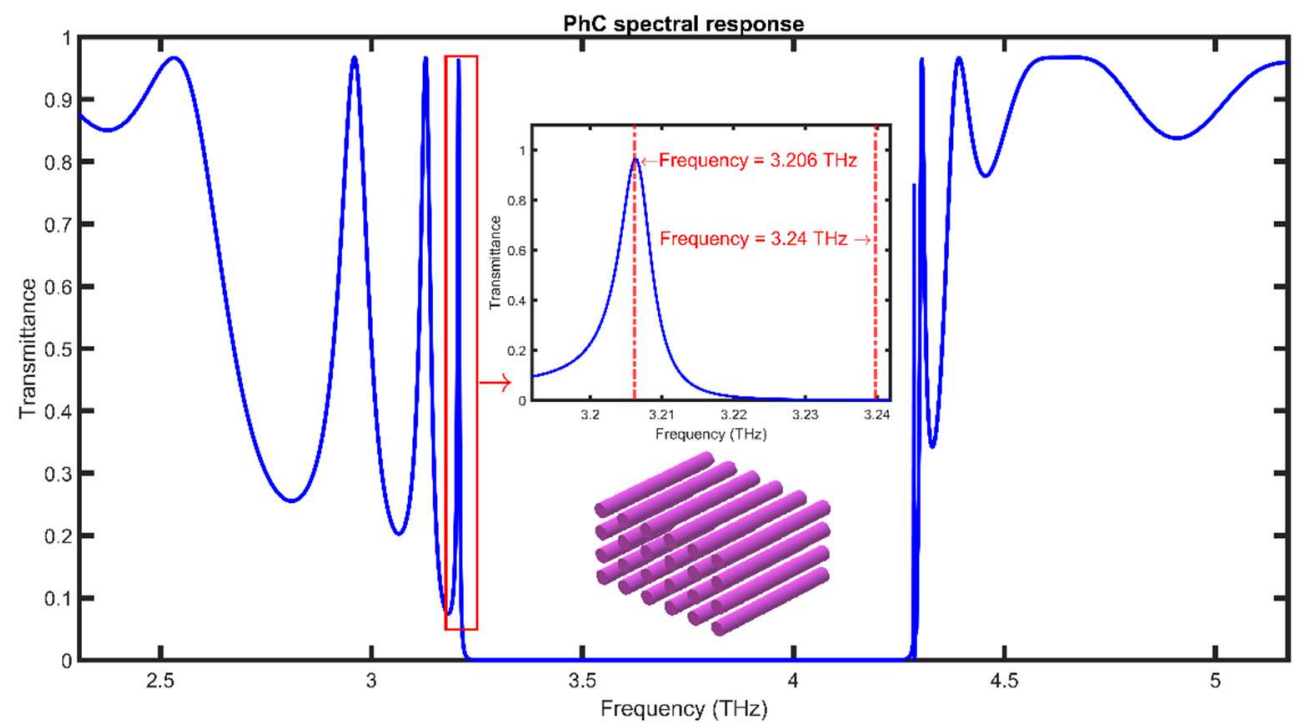

Fig. 3: The customized PhC spectral for transmittance response. The upper inset is the first left side of the bandgap (with a frequency peak point of $3.206 \mathrm{THz}$ ) and the lower inset is the schematic of the PhC. We have shown the left boundary of the PhC bandgap at $3.24 \mathrm{THz}$ in the upper inset with the dasheddotted vertical line.

Fig. 4 shows the magnitude of the transmission coefficient versus temporal harmonic indices corresponding to the TM polarization for the normal incident. Fig. 4(a) shows time-varying down- converter from $f_{0}$ to $f_{0}-f_{m}$ and Fig. 4(b) shows time-dependent up-converter from fundamental frequency to the first harmonic frequency $\left(f_{0}\right.$ to $\left.f_{0}+f_{m}\right)$. The backward slab has the same parameter as the forward slab except for the rhythm of permittivity variation that can be shown with $\varepsilon_{b}(t)=\varepsilon_{r b}\left(1-\delta_{\varepsilon} f_{m} t\right)$, where $\varepsilon_{r f}=\varepsilon_{r b}, d_{f}=d_{b}$. The modulation depth of $\delta_{\varepsilon}$ is equal for both down- and up-converter metasurfaces.
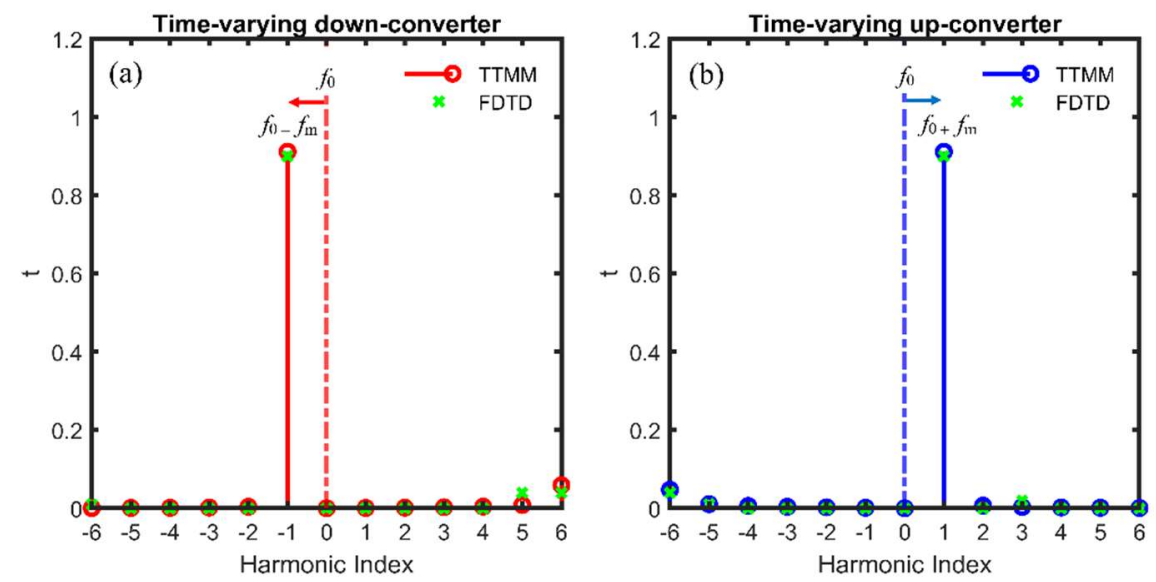

Fig. 4: The magnitude of the transmission coefficient versus temporal harmonic indices for time-varying (a) down- converter and (b) up-converter metasurface with red- and blue- shift, respectively. All figures have TM polarization with the linear time-varying permittivity of $\varepsilon_{\{f, b\}}(t)=\varepsilon_{\{r f, r b\}}\left(1 \pm \delta_{\varepsilon} f_{m} t\right)$ and 
permeability of $\mu_{\{f, b\}}(t)=\mu_{\{r f, r b\}}\left(1 \pm \delta_{\mu} f_{m} t\right)$. The \pm is for down- and up-convert, respectively. The green crossed signs are FDTD verifications.

In Fig. 4, we used time-dependent permeability to prevent reflection due to time-varying local impedance. The formulation of permeability for down- and up-converted metasurfaces have been considered as $\mu_{f}(t)=\mu_{r f}\left(1+\delta_{\mu} f_{m} t\right)$ and $\mu_{b}(t)=\mu_{r b}\left(1-\delta_{\mu} f_{m} t\right)$, respectively. Local impedance mismatch in time boundary yields local reflection (Xiao et al. 2014). So, by considering identical time modulation of dielectric permittivity and magnetic permeability, i.e. $\delta_{\varepsilon}=\delta_{\mu}$, the slab is designed in time equilibrium and $\eta=\sqrt{\mu(t) / \varepsilon(t)}=\eta_{0} \eta_{r}$ will be constant. In our design, we considered $\mu_{r f}=\mu_{r b}$ to have identical metasurface on the forward and backward sides. Here, we have assumed $\varepsilon_{r f}=\mu_{r f}$ for convenience.

Practical implementation of the time-varying permittivity and permeability slab represents an interesting topic of research that has presented in some scenarios for weak modulation depth (Taravati 2018; Saadoun and Engheta 1992; Lira et al. 2012; Qin et al. 2014; Martínez-Romero et al. 2016). We think that combining these scenarios with graphene-based structures could be a promising way to achieve time-dependent permittivity and permeability with greater modulation depth.

\section{Discussion}

As depicted in the simulation of the metasurfaces, by injection of the incident plane wave in the frequency of $f_{0}=3.24 \mathrm{THz}$, which is in the PhC bandgap sufficiently, the upper metasurface down-convert it from $f_{0}$ to $f_{0}-f_{m}$, which is equal to $3.206 \mathrm{THz}$, and can be passed with high transmission coefficient through $\mathrm{PhC}$ reflector. The transmitted wave at the frequency of the $f_{0}-f_{m}$, up-convert to the original frequency in the second metasurface. In time-reversed excitation, if we inject the backward wave with the frequency of $f_{0}$, the second metasurface up-converts the wave to the $f_{0}+f_{m}$, which is in the PhC bandgap and reflects from the PhC reflector. The reflected wave interacts with the second metasurface again to experience new up-conversion from $f_{0}+f_{m}$ to $f_{0}+2 f_{m}$. For $f_{0}+f_{m}$ and $f_{0}-f_{m}$ injection from the backward line, we have an up-convert state to $f_{0}+2 f_{m}$ and $f_{0}$, respectively, that are in the $\mathrm{PhC}$ bandgap and reflected again.

There is need to employ bulky component of $\mathrm{PhC}$ in our setup because such a setup cannot do the same thing as we presented here only upon introducing time modulation metasurfaces.

The modulation frequency is assumed to be $34 \mathrm{GHz}$ while the operating one is $3.24 \mathrm{THz}$. This will guarantee that the system is in the adiabatic regime of modulation where $f_{m} / f_{0} \ll 1$, which is a well-established assumption in most time-modulated works (Suchowski et al. 2014).

The nonreciprocity has been investigated under normal incidence. If we change the incident angle, we will have another distribution of temporal harmonics rather than Fig. 4, that is not suitable for our proposed application.

We can use this method of adding active metasurfaces to any asymmetrical one-way transmitters like that proposed in (Khorrami et al. 2020) to prevent any further design and immigrate from asymmetric to the nonreciprocal structure quickly. Designing the integrated structure using guided-mode resonance grating with time-varying metasurfaces is another idea to develop ideal isolators. Also, we can redesign the proposed $\mathrm{PhC}$ in the form of hole-based $\mathrm{PhC}$ instead of rod-based to easily integrate the nonreciprocal structure with two metasurfaces slabs.

\section{Summary}

We developed a numerical method to simulate multilayer metasurfaces considering timevarying permittivity and permeability based on the scattering matrix. Then, we proposed an emulation design method to combine $\mathrm{PhC}$ reflector with time-modulated metasurfaces resulting in a nonreciprocal isolator. CA method was used to quickly and accurately optimize the 
parameters. We demonstrated that the time-reversal symmetry of every asymmetrical structure can be broken using this method, although pure time-varying metasurfaces cannot prepare full nonreciprocity alone. Results show good numerical outputs and enough guard band to safely design the structure. Finally, we proposed some new ideas to continue this promising research in the future. It is impossible at the present time to realize real thin metamaterial slab with modulated permittivity and permeability. Indeed, it is used only in simulation for emulating the time varying metasurfaces and estimate the performances of the devices based on them. Therefore, we believe that this is the beginning of discovering new areas of non-reciprocating isolators using a new type of equilibrated material.

Disclosures. The authors declare no conflicts of interest.

Code availability: All codes developed in this work will be made available from the corresponding author upon reasonable request.

\section{References}

1. Caloz, C., Deck-Léger, Z.: Spacetime Metamaterials - Part I: General Concepts. IEEE Trans. Antennas Propag. 68, 1569-1582, (2020).

2. Chamanara, N., Vahabzadeh, Y., Caloz, C.: Simultaneous Control of the Spatial and Temporal Spectra of Light with Space-Time Varying Metasurfaces. IEEE Trans. Antennas Propag. 67, 2430-2441 (2019).

3. Taravati, S., Eleftheriades, G. V.: Generalized Space-Time-Periodic Diffraction Gratings: Theory and Applications. Phys. Rev. Applied 12, 024026 (2019).

4. Pendry, J. B.: Time Reversal and Negative Refraction. Science, 322, 71-73 (2008).

5. Cui, T., Bai, B. F., Sun, H.-B.: Tunable Metasurfaces Based on Active Materials. Adv. Funct. Mater. 29, 1806692 (2019).

6. Pacheco-Peña, V., Engheta, N.: Temporal aiming. Light Sci. Appl. 9, 129 (2020).

7. Zang, J.W., Correas-Serrano, D. J., Do, T.S., Liu, X., Alvarez-Melcon, A., Gomez-Diaz, J.S.: Nonreciprocal Wavefront Engineering with Time-Modulated Gradient Metasurfaces. Phys. Rev. Applied 11, 054054 (2019).

8. Taravati, S.: “Aperiodic space-time modulation for pure frequency mixing," Phys. Rev. B 97, 115131 (2018).

9. Shaltout, A., Kildishev, A., Shalaev, V.: Time-varying metasurfaces and Lorentz non-reciprocity. Opt. Mater. Express 5, 2459-2467 (2015).

10. Wu, Z., Grbic, A.: Serrodyne Frequency Translation Using Time-Modulated Metasurfaces. IEEE Trans. Antennas Propag. 68, 1599-1606 (2020).

11. Xiao, Y., Maywar, D. N., Agrawal, G. P.: Reflection and transmission of electromagnetic waves at a temporal boundary. Opt. Lett. 39, 574-577 (2014).

12. Chegnizadeh, M., Memarian, M., Mehrany, K.: Non-reciprocity using quadrature-phase time-varying slab resonators. J. Opt. Soc. Am. B 37, 88-97 (2020).

13. Sedeh, H. B., Salary, M. M., Mosallaei, H.: Topological Space-Time Photonic Transitions in AngularMomentum-Biased Metasurfaces. Adv. Opt. Mater. 8, 2000075 (2020).

14. Ba, C., Huang, L., Liu, W., Li, S., Ling, Y., Li, H.: Narrow-band and high-contrast asymmetric transmission based on metal-metal-metal asymmetric gratings. Opt. Express 27, 25107-25118 (2019).

15. Tang, B., Li, Z., Liu, Z., Callewaert F., Aydin, K.: Broadband asymmetric light transmission through tapered metallic gratings at visible frequencies. Sci. Rep. 6, 1-7 (2016).

16. Ling, Y., Huang, L., Hong, W., Liu, T., Sun, Y., Luan, J., Yuan, G.: Asymmetric optical transmission based on unidirectional excitation of surface plasmon polaritons in gradient metasurface. Opt. Express 25, 13648-13658 (2017)

17. Jalas, D., Petrov, A., Eich, M., Freude, W., Fan, S., Yu, Z., Baets, R., Popović, M., Melloni, A., Joannopoulos, J. D., Vanwolleghem, M., Doerr, C. R., Renner, H.: What is -and what is not- an optical isolator. Nat. Photon 7, 579-582 (2013).

18. Khorrami, Y., Fathi, D., Rumpf, R. C.: Fast optimal design of optical components using the cultural algorithm. Opt. Express 28, 15954-15968 (2020).

19. Xu, T., Lezec, H. J.: Visible-frequency asymmetric transmission devices incorporating a hyperbolic metamaterial. Nat. Commun. 5, 1-7 (2014).

20. Li, S., Huang, L-r., Ling, Y-h., Liu, W-b., Ba, C-f., Li, H-h.: High-performance asymmetric optical transmission based on coupled complementary subwavelength gratings. Sci. Rep. 9, 17117 (2019).

21. Stolarek, M., Yavorskiy, D., Kotyński, R., Rodríguez, C. J. Z., Łusakowski, J., Szoplik, T.: Asymmetric transmission of terahertz radiation through a double grating. Opt. Lett. 38, 839-841 (2013).

22. Cakmakyapan, S., Serebryannikov, A. E., Caglayan, H., Ozbay, E.: One-way transmission through the subwavelength slit in nonsymmetric metallic gratings. Opt. Lett. 35, 2597-2599 (2010). 
23. Zhu, R., Wu, X., Hou, Y., Zheng, G., Zhu, J., Gao, F., Broadband Asymmetric Light Transmission at Metal/Dielectric Composite Grating. Sci. Rep. 8, 1-9 (2018).

24. Xu, P., Lv, X., Chen, J., Li, Y., Qian, J., Chen, Z., Qi, J., Sun, Q., Xu, J.: Dichroic Optical Diode Transmission in Two Dislocated Parallel Metallic Gratings. Nanoscale Res. Lett. 13, 1-8 (2018).

25. Serebryannikov, A. E., Colak, E., Magath, T., Ozbay, E.: Two types of single-beam deflection and asymmetric transmission in photonic structures without interface corrugations. J. Opt. Soc. Am. A 33, 2450-2458 (2016).

26. Maystre, D.: Photonic crystal diffraction gratings. Opt. Express 8, 209-216 (2001).

27. Blankrot, B., Heitzinger, C.: Design of aperiodic demultiplexers and optical diodes by optimizing photonic crystals. OSA Continuum. 2, 2244-2252 (2019).

28. Bor, E., Turduev, M., Yasa, U. G., Kurt, H., Staliunas, K.: Asymmetric light transmission effect based on an evolutionary optimized semi-Dirac cone dispersion photonic structure. Phys. Rev. B 98, 245112 (2018).

29. Salary, M. M., Farazi, S., Mosallaei, H.: A Dynamically Modulated All-Dielectric Metasurface Doublet for Directional Harmonic Generation and Manipulation in Transmission. Adv. Opt. Mater. 7, 1900843 (2019).

30. Yuanhang, C., Xiaoting, W., Qinghai, S., Yabei, Z., Shumin, X.: Tunable optical metasurfaces enabled by multiple modulation mechanisms. Nanophotonics, 9, 4407-4431 (2020).

31. Salary, M. M., Jafar-Zanjani, S., Mosallaei, H.: Electrically Tunable Harmonics in Time-modulated Metasurfaces for Wavefront Engineering. New J. Phys. 20, 123023 (2018).

32. Shaltout, A. M., Shalaev, V. M., Brongersma, M. L.: Spatiotemporal light control with active metasurfaces. Science 364 (2019).

33. Khorrami, Y., Fathi, D., Rumpf, R. C.: Guided-mode resonance filter optimal inverse design using one- and twodimensional grating. J. Opt. Soc. Am. B 37, 425-432 (2020).

34. Salary, M. M., Jafar-Zanjani, S., Mosallaei, H.: Time-varying metamaterials based on graphene-wrapped microwires: Modeling and potential applications. Phys. Rev. B 97, 115421 (2018).

35. Hao, J., Zhou, L.:Electromagnetic wave scatterings by anisotropic metamaterials: Generalized $4 \times 4$ transfermatrix method. Phys. Rev. B 77, 094201 (2008).

36. Park, J. h., Kang, Ju-H., Kim, S. J., Liu, X., Brongersma, M. L.: Dynamic Reflection Phase and Polarization Control in Metasurfaces. Nano Letters 17, 407-413 (2017).

37. Rumpf, R. C.: Improved Formulation of Scattering Matrices for Semi-Analytical Methods That Is Consistent with Convention. Progress in Electromagnetics Research B 35, 241-261 (2011).

38. Stewart, S. A., Smy, T. J., Gupta, S.: Finite-Difference Time-Domain (FDTD) Modelling of Space-Time Modulated Metasurfaces. IEEE Trans. Antennas Propag. 66, 281-292 (2018).

39. Inampudi, S., Salary, M. M., Jafar-Zanjani, S., Mosallaei, H.: Rigorous space-time coupled-wave analysis for patterned surfaces with temporal permittivity modulation. Opt. Mater. Express 9, 162-182 (2019).

40. Phare, C. T., Lee, Y.-H. D., Cardenas, J., Lipson, M.: Graphene electro-optic modulator with 30 GHz bandwidth. Nat. Photonics 9, 511 (2015).

41. Xiao, Y., Maywar, D. N., Agrawal, G. P.: Reflection and transmission of electromagnetic waves at a temporal boundary. Opt. Lett. 39, 574-577 (2014).

42. Taravati, S.: Giant linear nonreciprocity, zero reflection, and zero band gap in equilibrated space-time-varying media. Phys. Rev. Appl. 9, 064012 (2018).

43. Saadoun, M. M. I., Engheta, N.: A reciprocal phase shifter using novel pseudochiral or $\omega$ medium. Microwave Opt. Technol. Lett. 5, 184 (1992).

44. Lira, H., Yu, Z., Fan, S., Lipson, M.: Electrically Driven Nonreciprocity Induced by Interband Photonic Transition on a Silicon Chip. Phys. Rev. Lett. 109, 033901 (2012).

45. Qin, S., Xu, Q., Wang, Y. E.: Nonreciprocal components with distributedly modulated capacitors. IEEE Trans. Microwave Theory Tech. 62, 2260 (2014).

46. Martínez-Romero, J. S., Becerra-Fuentes, O. M., Halevi, P.: Temporal photonic crystals with modulations of both permittivity and permeability. Phys. Rev. A 93, 063813 (2016).

47. Suchowski, H., Porat, G., Arie, A.: Adiabatic processes in frequency conversion. Laser Photonics Rev. 8, 333367 (2014). 